\title{
COMPSAC 2015 Symposium on
}

\section{Technologies and Applications of the Internet (TAIN) Message from TAIN Organizing Committee}

It is a pleasure to welcome you to COMPSAC 2015 Symposium on Technologies and Applications of the Internet (TAIN). This year, first time in its history, COMPSAC is organized as a series of symposiums under the main theme of "Mobile and Cloud Systems - Challenges and Applications". TAIN Symposium providing a forum to share latest innovations on Internet technologies and applications perfectly matches with the main theme. Topics of interest for TAIN include all types of networks with their architectures and applications as well as their management, performance, and security. Moreover, TAIN Symposium addresses Internet of things (IoT), machine-to-machine (M2M) and peer-to-peer (P2P) communications, content distribution networks (CDN) and also emerging network technologies such as software defined networks (SDN). A vast array of challenges for Internet technologies and applications and proposals for solutions will be discussed in TAIN Symposium.

This year, TAIN received 27 full submissions. Each submission underwent a rigorous doubleblind review process and was discussed by the program chairs during the PC meeting, which took place in Shanghai, China, in March 2015. As the result of this process, we accepted $18.5 \%$ of the submissions as regular papers and $26 \%$ of the submissions as short papers for inclusion into this year's COMPSAC proceedings as TAIN Symposium papers, published by IEEE CS. These papers will be presented in three regular sessions of TAIN Symposium. We would like to take this opportunity to thank the authors, who submitted their valuable research results to the TAIN Symposium and the members of the TAIN Program Committee and external reviewers, who contributed to the success of the program assembled for TAIN 2015.

We would like to express our gratitude to Carl Chang, Standing Committee Chair of COMPSAC, who is truly the backbone of COMPSAC. We extend our sincere thanks to William (Cheng-Chung) Chu (Steering Committee Chair) and David Alan Grier, Sheikh Iqbal Ahamed, and Shih-Chiin Lee (General Chairs) for their ongoing support and contributions. We wish to especially thank Gang Huang, Ivica Crnkovic, and Pao-Ann Hsiung (Program Chairs-in-Chief) for their coordination and putting everything together for a great COMPSAC program. Our thanks will also be for all the chairs and the organizing committee for their hard work.

We hope that you will find the symposium both valuable and productive.

\section{TAIN Program Co-Chairs}

Tugkan Tuglular, Izmir Institute of Technology, Turkey

Ying Cai, Iowa State University, USA

\section{TAIN General Chair}

Schahram Dustdar, Vienna University of Technology, Austria

\section{Standing Committee Representative}

Katsuyuki Yamazaki, Nagaoka University of Technology, Japan 